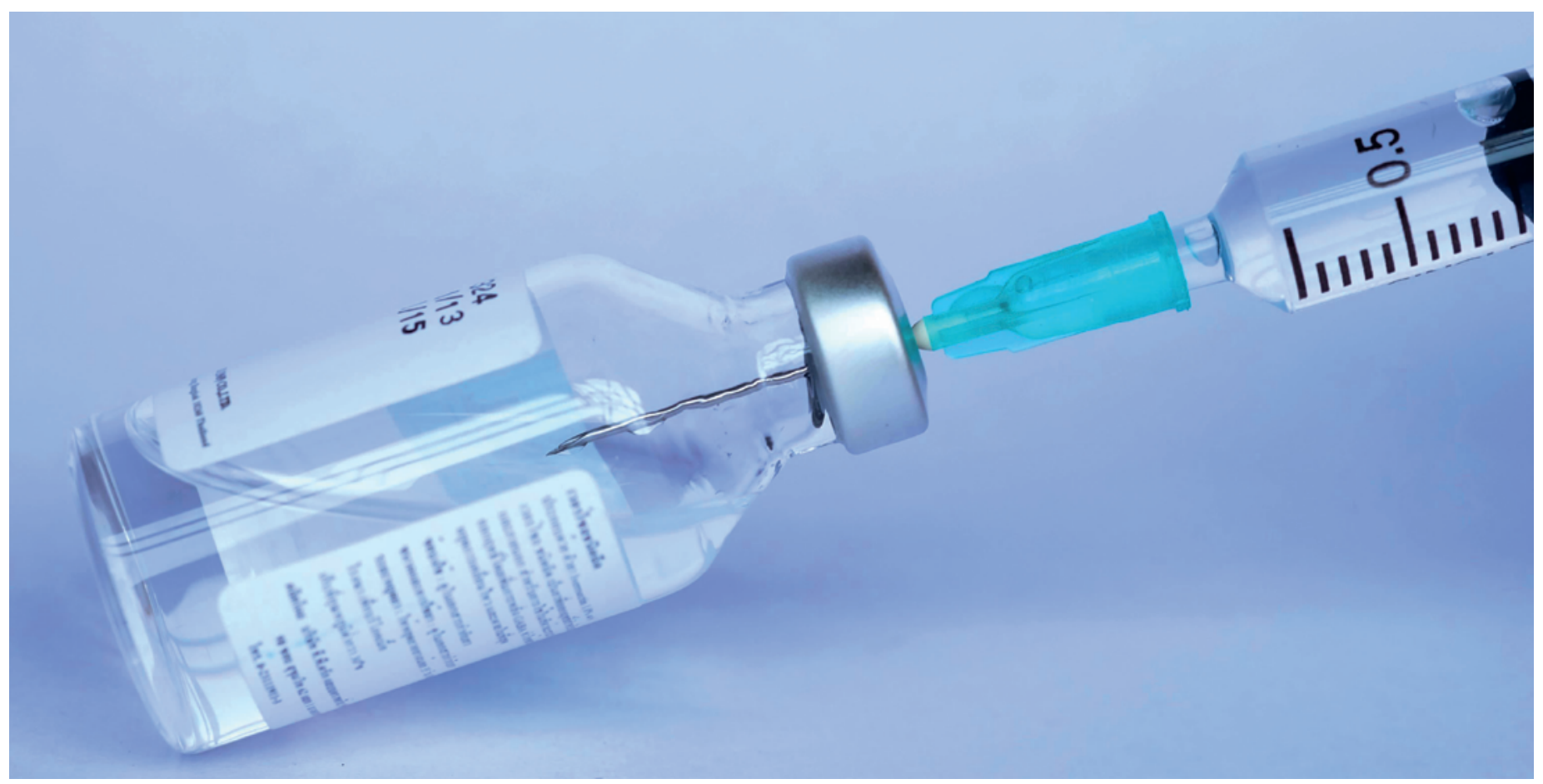

\title{
Versorgungssicherheit mit Impfstoffen
}

\section{Prof. Dr. med. Christoph Berger ${ }^{a}$, Dr. med. Carlos B. Quinto ${ }^{b}$, unter Mitarbeit von: Barbara Weilc}

a Präsident Eidgenössische Kommission für Impffragen (EKIF), Leiter Abteilung Infektiologie und Spitalhygiene Universitäts-Kinderspital Zürich;

${ }^{b}$ Mitglied des FMH-Zentralvorstandes, Departementsverantwortlicher Public Health, Gesundheitsberufe und Heilmittel; ${ }^{\circ}$ Leiterin Abteilung Public Health FMH; Linda Hadorn, wiss. Mitarbeiterin Abteilung Public Health FMH

Sollen Impfungen die Bevölkerung optimal schützen, müssen folgende Bedingungen erfüllt sein: Die Impfstoffe sind national zugelassen, die Versorgung mit ebendiesen ist gewährleistet, die Impfungen werden offiziell empfohlen sowie durch die obligatorische Krankenkasse vergütet, und die Ärzteschaft setzt die Impfempfehlungen um. Alle diese Vorgaben einzuhalten ist offensichtlich nicht einfach.

Impfungen sind in der Bekämpfung von Infektionskrankheiten weltweit ein essentieller und erfolgreicher Public-Health-Ansatz. Sie stellen eine wirksame und kostengünstige Massnahme zum Schutz von Individuum und Gesellschaft dar. Die Versorgungssicherheit mit Impfstoffen ist die Schlüsselkomponente für die erfolgreiche Umsetzung dieser Präventionsmassnahme. In letzter Zeit sind in der Schweiz immer häufiger und über längere Zeit die notwendigen Impfstoffe nicht verfügbar. Dies hat bei den impfenden Ärztinnen und Ärzten, in kantonalen Gesundheitsämtern wie auch bei betroffenen Fachgesellschaften verschiedent- lich Anlass zur Sorge gegeben. Ziel dieses Artikels ist, das Fachpublikum über Hintergründe der zeitweise mangelnden Impfstoffversorgung sowie die geplanten Schritte zur Entschärfung des Problems zu informieren.

\section{Ausgangslage}

Seit 1963 publiziert das Bundesamt für Gesundheit (BAG) Impfempfehlungen und passt diese laufend den aktuellen Gegebenheiten an. Da Impfungen immer wichtiger werden, initiierte Bundesrat Pascal Couchepin 
Impfstoff-Versorgung:

Organisationen und ihre Aufgaben bei Liefer- und Versorgungsengpässen

Bundesamt für wirtschaftliche Landesversorgung BWL: Meldestelle für Versorgungsengpässe, die voraussichtlich mehr als 14 Tage dauern, sowie Koordination der Pflichtlager für alle empfohlenen Impfstoffe (www.bwl.admin.ch/meldestelle www.bwl.admin.ch/bureau-de-notification - www.bwl.admin.ch/ centro-di-notifica - www.bwl.admin.ch/reporting-office).

Swissmedic: Gesuche um befristetes Inverkehrbringen eines in der Schweiz zugelassenen Impf-Präparates in ausländischer Aufmachung, Sonderbewilligungen zum Import von Impfstoffen, die in der Schweiz nicht zugelassen sind, sowie Bekanntgabe der Länder, aus denen ein in der Schweiz zugelassenes Arzneimittel eingeführt werden kann.

Bundesamt für Gesundheit BAG: Zusammen mit der EKIF (Eidgenössische Kommission für Impffragen, s. auch u.) zuständig für Anpassungen der Impfempfehlungen bei Versorgungsengpässen (basierend auf dem EpG) und deren Publikation sowie Sicherstellung der Vergütung von Ersatzimpfstoffen.

Eidgenössische Kommission für Impffragen (EKIF): Erarbeitung und Publikation von provisorischen Empfehlungen während Versorgungsengpässen sowie Klärung der Kostenrückerstattungsfrage.

2004 die unabhängige Eidgenössische Kommission für Impffragen (EKIF). Nebst der fachlichen Einschätzung der EKIF und des BAG müssen Impfempfehlungen die Gegebenheiten des nationalen Gesundheitssystems berücksichtigen. Dazu gehören unter anderem die medizinische Grundversorgung (dort werden die meisten Impfungen verabreicht), Vorsorgeuntersuchungen (Pädiatrie, Gynäkologie usw.) sowie die Strukturen der Kostenübernahmeregelungen. Auf der Basis des Bundesgesetzes über die Bekämpfung übertragbarer Krankheiten des Menschen (EpG) entwickelte das BAG zudem die Nationale Strategie zu Impfungen (NSI), die 2017 genehmigt wurde. Die Strategie will, dass die Impfempfehlungen koordiniert und effizient verbreitet und umgesetzt werden.

\section{In letzter Zeit sind in der Schweiz immer häufiger und über längere Zeit die notwendigen Impfstoffe nicht verfügbar.}

Die Thematik der ausreichenden Versorgung mit Impfstoffen erfährt grosse Aufmerksamkeit und ist nicht ausschliesslich ein schweizerisches Problem. Aufgrund der Marktaufteilung bekunden viele Länder Schwierigkeiten, genügend Impfstoffe zu erhalten. Laut einer Umfrage [1] der WHO in Europa aus dem Jahr 2015 meldeten 77\% der befragten Länder [2], dass sie im Jahr der Befragung mindestens einen Versorgungsengpass bei Impfstoffen erlebt hatten. In diesem Zusammenhang gilt es zu unterscheiden zwischen Lieferengpässen und Versorgungsengpässen. Bei Lieferengpässen können bestimmte Produkte nicht in ein oder mehrere Länder geliefert werden. Bei Versorgungsengpässen fehlen bestimmte Produkte weltweit.

Bislang führten in der Schweiz die Engpässe glücklicherweise noch zu keinen gravierenden Public-HealthProblemen. Es kam aber zu erheblichen Schwierigkeiten bei Impfaktionen, zu zusätzlichen Bemühungen, Aufwand und Ärger bei engagierten Grundversorgenden. Die Nichtverfügbarkeit von Impfstoffen erschwert die Durchführung der im Impfplan festgehaltenen Empfehlungen und führt zu zusätzlichen Konsultationen. Ausserdem kommt es zum Einsatz von Kombinationspräparaten anstelle monovalenter Impfstoffe oder zu zusätzlichen Impfungen, was Mehrkosten generiert. Engpässe stellen zudem die Glaubwürdigkeit von Impfkampagnen in Frage und verunsichern Impfwillige, was die Ärzteschaft bei der Umsetzung der Impfempfehlungen zu spüren bekommt. Die Verunsicherung wiederum hat Auswirkungen auf die Impfbereitschaft und Durchimpfung im Allgemeinen.

Die Entwicklung zeigt, dass die Liefer- und Versorgungsengpässe und deren Ursachen (noch) nicht gelöst sind. Im Gegenteil ist zu erwarten, dass in der Schweiz weiterhin Lieferengpässe bei Impfstoffen auftreten werden und weltweit die Versorgungsengpässe zunehmen. Gefährliche Lücken in der Grundimmunisierung sind möglich. Deren Auswirkungen könnten sich erst mit einer unbestimmten Latenz manifestieren, dafür dann aber umso unkontrollierbarer und mit grösseren Gesundheits- und Kostenfolgen.

\section{Ursachensuche}

Die Ursachen der Problematik sind vielschichtig. Hauptsächlich verantwortlich sind die komplexe Produktion, die Eintrittshürden in den Schweizer Markt, die Übernahmen unter Pharmakonzernen und die damit einhergehende Konzentration der Anbieterfirmen. Weiter verantwortlich sind die steigende Nachfrage nach Impfungen weltweit wie auch uneinheitliche und unterschiedlich restriktive Zulassungsprozesse in verschiedenen Ländern und die Thematik der Kostenübernahme.

Die Komplexität in der Produktion und der Verteilung von Impfstoffen hat in den letzten Jahren kontinuierlich zugenommen, weil sich die Produktionskette innerhalb eines hochregulierten Marktumfeldes bewegt. Folgen der Globalisierung und steigender Preisdruck wirken sich negativ auf die Versorgungssicherheit aus. So verlagern Wirkstoff verarbeitende Unternehmen 
ihre Standorte ins Ausland, mit dem Resultat, dass heute $80 \%$ der Impfstoffe in China und Indien hergestellt werden. Weiter sind die Unternehmen nicht verpflichtet, für Patienten wichtige Mittel auf dem Markt zu halten, die keinen Gewinn abwerfen. In Krisensituationen spitzt sich diese Ausgangslage noch zu und erhöht die Gefahr einer Unterversorgung.

Das hochregulierte Marktumfeld ist eine Sache, die weltweit steigende Nachfrage eine andere. In der Schweiz erhalten neben Säuglingen und Kleinkindern heute auch Jugendliche, jede Schwangere und enge Angehörige von jungen Säuglingen im Rahmen einer Extraauffrischung eine dTpa-Kombinationsimpfung. Dies hat den Bedarf an Impfstoffen ansteigen lassen. Die Nachfrage nach Impfstoffen wird aber nicht nur in der Schweiz, sondern weltweit immer grösser. Auf die steigende Nachfrage kann jedoch nicht kurzfristig reagiert werden, denn die Herstellung von Impfstoffen ist aufwendig. Je nach Produkt nimmt dies bis zu 18 Monate in Anspruch. Bei schnell steigender Nachfrage kommt die Produktion also kaum nach.

\section{Gefährliche Lücken in der Grundimmunisie- rung sind möglich.}

Auch technische Pannen oder Qualitätsmängel können zu Versorgungsengpässen führen, denn die dadurch notwendige (Neu)-Produktion lässt sich nicht in der Zeit gewährleisten, die für eine lückenlose Versorgung weltweit notwendig ist. Diese Problematik könnte über einen diversen Markt aufgefangen werden. In der Folge von Konzentrationsprozessen wird dieser aber im Gegenteil immer kleiner. Fällt bei einem Unternehmen das Produkt weg, schwinden somit auch die Möglichkeiten, auf einen Konkurrenzanbieter zu wechseln. Lagerung und Vertrieb werden an wenigen Standorten konzentriert und damit die dezentralen Versorgungsstrukturen reduziert. Bei einigen Impfstoffen hat dies zu einer Oligo- oder sogar Monopolsituation geführt. Betriebswirtschaftlich ist diese Entwicklung durchaus nachvollziehbar, für die Versorgungssicherheit und die Preise ist sie ein klarer Nachteil.

Die Impfstoffversorgung in der Schweiz ist aus drei Gründen besonders anfällig für Engpässe. Erstens, weil sie vollumfänglich von international tätigen Herstellungsfirmen abhängig ist. Zweitens sind europäisch zugelassene Impfstoffe, welche das sorgfältige und aufwendige Zulassungsverfahren - durchgeführt von der Europäischen Arzneimittel-Agentur (EMA) - bestanden haben, in der Schweiz nicht zugelassen. Hierzulande ist für die Zulassung von Medikamenten das Schweizerische Heilmittelinstitut (Swissmedic) zu- ständig. Antragstellende müssen bei swissmedic erneut eine umfangreiche Dokumentation einreichen und Wirksamkeit, Qualität, Sicherheit sowie Identität, Reinheit und Wirkstoffgehalt ein weiteres Mal belegen. Ein solches Verfahren dauert rund 330 Tage. Seit Anfang 2013 existiert mit Voranmeldung ein beschleunigtes Verfahren. Doch auch hier ist mit einer Dauer von 264 Tagen sowie mit höheren Gebühren zu rechnen. Dieses Vorgehen ist mit viel Aufwand und Kosten verbunden und bei einem negativen Entscheid werden die Antragstellenden vor Probleme gestellt werden. Das veranlasst sie dazu, die Zulassungsanfrage zurückzuziehen oder gar nicht erst einzureichen. Denn das Schweizer Marktvolumen hat vergleichsweise wenig Bedeutung. Das führt dazu, dass in der Schweiz heute rund 30 Impfstoffe weniger zugelassen sind als in den umliegenden Ländern. Konkrete Aussagen über die Gründe von nicht erfolgten Zulassungen zu erhalten ist schwierig, da Informationen zum konkreten Zulassungsverfahren vertraulich sind. Mit dem revidierten Heilmittelgesetz, das voraussichtlich 2019 in Kraft tritt, würde aber die rechtliche Grundlage geschaffen, dass Swissmedic transparenter informieren könnte. Drittens ist Versorgung von Impfstoffen in der Schweiz anfällig, weil sie über den freien Markt erfolgt und dezentral ist. Das heisst, es erfolgt keine zentrale Bestellung. Die Impfstoffe werden von einer Vielzahl von Apotheken, Arztpraxen und Spitälern und je nach Kanton und Impfung auch von schulärztlichen Diensten oder kantonalen Stellen bei den jeweiligen Herstellungsfirmen oder im Grosshandel bestellt. Der Bund hat also zurzeit im Bestellprozess keine Aufgabe, er kommt nur subsidiär, d.h. im Krisenfall, zum Zug. Zusätzlich ist die Problematik der Kostenübernahme durch die OKP zu erwähnen. Eine Kostenübernahme wird verweigert, wenn entweder die Impfstoffe von Swissmedic nicht zugelassen sind oder wenn sie zwar zugelassen sind, aber vom BAG (KUV) und EDI nicht auf die Spezialitätenliste gesetzt wurden, weil die betroffenen Impfstoffe die WZW Kriterien nicht erfüllen (aktuelle Beispiele Zostavax, Rotarix). Diese Problematik ist darauf zurückzuführen, dass die Schweiz ein Krankenversicherungsgesetz hat, dieses jedoch kein Präventionsgesetz ist.

\section{Lösungen}

Kurzfristig müssen Ärztinnen und Ärzte bei Engpässen derzeit (noch) auf Reserven in lokalen Kliniken und Spitälern oder auf die Vorräte der Grosshandelsfirmen zurückgreifen - oder selber Reserven anlegen. Eine andere Möglichkeit ist, bei Engpässen von Kombinations- oder Einzelimpfstoffen diese durch andere, nicht optimal passende (fehlende oder überzählige 
Komponenten) Kombinationsimpfstoffe (Ersatzempfehlungen) zu ersetzen.

Eine aufwändige und im Alltag leider nicht praktikable Option für Ärztinnen und Ärzte ist, bei Impfstoffengpässen auf den analogen Impfstoff eines anderen Herstellers im Ausland auszuweichen. Bedingung ist, dass sie die betreffenden Alternativen kennen. Sind diese Alternativen in der Schweiz nicht verfügbar oder nicht zugelassen, kann eine Ärztin / ein Arzt einen in der Schweiz nicht zugelassenen gleichwertigen Impfstoff aus dem Ausland importieren. Für die Einfuhr von Impfstoffen benötigt eine Medizinalperson vorgängig eine Sonder- und Einfuhrbewilligung von Swissmedic (www.swissmedic.ch/sonderbewilligungen). Die Einzeleinfuhrbewilligung für die beantragenden Ärztinnen und Ärzte sowie die Spitäler ist kostenpflichtig und mit administrativem Aufwand verbunden, kommt jedoch den Patienten und Patientinnen zugute. Die Verantwortung für Einfuhr, Abgabe und Anwendung liegt vollumfänglich bei der behandelnden Ärztin, beim behandelnden Arzt. Diese sind verpflichtet, Patienten über den Arzneimitteleinsatz hinreichend aufklären, die Einwilligung einzuholen und zu dokumentieren. Die Patientin / der Patient ist auch darüber in Kenntnis setzen, dass diese alternative Anwendung grundsätzlich keine Vergütungspflicht der obligatorischen Krankenpflegeversicherung begründet. Dies bedeutet, dass die Patientin / der Patient vorgängig über die Vergütungssituation zu informieren ist. Für deren definitive Klärung sind die Patienten/Patientinnen an die Krankenversicherer zu verweisen; einerseits, da die Versicherungspolicen eine unübersichtliche Heterogenität aufweisen und andererseits Ärztinnen und Ärzten ab 1.1.2018 auf Grund der Zeitlimitationen für die Tarifpositionen Konsultation und Leistungen in Abwesenheit, welche auf Betreiben der Krankenversicherer vom Bundesrat im Rahmen des zweiten Tarifeingriffs eingeführt wurden, diese Zeit nicht mehr zur Verfügung steht. Da Fehlauskünfte am Telefon durch Krankenversicherer häufig sind, weil günstiges, unqualifiziertes Personal eingesetzt wird, ist bei relevanten Summen den Patienten / Patientinnen zu raten, die Information schriftlich beim Krankenversicherer einzufordern.

Schliesslich sind weitere Ansätze zu verfolgen, welche die Verfügbarkeit verschiedener gleichwertiger Impfstoffe (sofern existierend) für den Schweizer Markt proaktiv sicherstellen oder zumindest den Zugang dazu verbessern. Das Epidemiengesetz (Art. 44 Abs. 1 EpG) gibt dem Bundesrat die Kompetenz, die Versorgung der Bevölkerung mit den wichtigsten zur Bekämpfung übertragbarer Krankheiten geeigneten
Heilmitteln sicherzustellen, soweit er sie nicht durch Massnahmen nach dem Landesversorgungsgesetz gewährleisten kann. Weitere Massnahmen sind laut Aussage des Bundesamtes für Gesundheit in Planung, jedoch noch nicht spruchreif.

Um die Versorgungssicherheit längerfristig und aktiv zu gewährleisten, sind die Rahmenbedingungen auf allen Stufen (Forschung, Herstellung, Zulassung, Vertrieb) zu überprüfen und Verbesserungen zu evaluieren. Konkret geht es darum, zeitnahe Meldungen an die betroffenen Akteure (Frühwarnsysteme) einzurichten sowie Empfehlungen zur prioritären Anwendung der noch verfügbaren Impfstoffe zu erlassen. Ferner sind zentral geführte Pflichtlager im Aufbau.

\section{Die Komplexität in der Produktion und Vertei- lung von Impfstoffen hat in den letzten Jahren kontinuierlich zugenommen.}

Dank der Meldepflicht (Verordnung über die Meldestelle für lebenswichtige Humanarzneimittel, SR 531. 215.32, Art. 2 Abs. 1 Bst. d) erfahren die Gesundheitsbehörden des Bundes und der Kantone sowie die Leistungserbringenden sofort von allfälligen ImpfstoffLieferengpässen. Zu diesem Zweck hat das Bundesamt für wirtschaftliche Landesversorgung BWL ein Frühwarnsystem in Form einer Informations- und Koordinationsplattform «Meldestelle für lebenswichtige Humanarzneimittel» eingerichtet (https://www.bwl. admin.ch/meldestelle). Seit März 2016 werden dort alle Versorgungsengpässe von Impfstoffen, die voraussichtlich länger als 14 Tage dauern, unter Angabe der voraussichtlichen Dauer publiziert. Basierend auf die jeweilige Ausgangslage verfassen EKIF/BAG Ersatzempfehlungen. Über die Verfügbarkeit von Impfstoffen informieren InfoVac per Internet und via monatliches Bulletin sowie Herstellerunternehmen per Rundschreiben und im Internet.

Zusätzlich schreibt der Bundesrat vor, von ihm festgelegte lebenswichtige Güter vorrätig zu halten, u.a. Heilmittel (Lagerbestand für 4 Monate). Diese gesetzlich verankerten Pflichtlager sind eine Möglichkeit, die Versorgungssicherheit zu erhöhen. Länder mit Pflichtlager sind grundsätzlich weniger betroffen von Engpässen bei Impfstoffen, dies vor allem dann, wenn die Pflichtlager vom Staat selber geführt werden (beispielsweise Holland). Noch einen Schritt weiter gehen Länder wie Grossbritannien, wo die offiziell empfohlenen Impfstoffe von einer staatlichen Organisation zentral eingekauft, verwaltet und verteilt werden. In der Schweiz sind solche Systeme nicht möglich. Einerseits fehlen die gesetzlichen Grundlagen, andererseits 
auch Räume, Personal und die Infrastruktur für die Verteilung.

Seit dem 1. Oktober 2016 sind auch Impfstoffe, die im Schweizerischen Impfplan empfohlen werden, der Pflichtlagerhaltung unterstellt. Die Pflichtlager werden von privaten Unternehmen geführt und bezahlt. Die Einflussnahme des Bundes ist limitiert, denn die Pflichtwaren sind Eigentum dieser Firmen, welche für einen regelmässigen Warenumschlag sowie eine marktkonforme Qualität verantwortlich sind. Für die Pflichtlager gilt dabei das Prinzip der Gewinn- und Verlustlosigkeit. Im Bereich der Heilmittel ist die Helvecura-Genossenschaft die Selbsthilfeorganisation, welche die vom Bund übertragenen Aufgaben in der Pflichtlagerhaltung erfüllt. In Mangellagen kann der Bund die lagerhaltenden Firmen autorisieren, zur Versorgung des Marktes auf die Pflichtlager zurückzugreifen. Dies mittels eines Verordnungserlasses zur Pflichtlagerfreigabe durch das Eidgenössische Departement für Wirtschaft, Bildung und Forschung (WBF). Zu erwähnen ist, dass die im Herbst 2016 eingeführten Pflichtlager für Impfstoffe nur dürftig gefüllt oder teilweise noch immer leer sind aufgrund der nach wie vor angespannten Versorgungslage.

\section{Auch technische Pannen oder Qualitätsmängel können zu Versorgungsengpässen führen.}

Ein weiterer Teil der Lösung sind die Patientinnen und Patienten selber. Muss bei Impfstoffengpässen der Impfplan bei Konsultationen verändert werden, geht es darum, Patientinnen und Patienten gut zu informieren, damit sie die Änderung nachvollziehen können und die Konsequenzen (beispielsweise Zusatzkosten) kennen. Diese Klärungen sind jedoch zeitaufwändig und müssen auch entsprechend über die Tarifstruktur abgegolten werden. Denn Ärztinnen und Ärzte müssen hier Konsequenzen tragen, die sie selber nicht verursacht haben.

\section{Schlussfolgerungen}

Um die Versorgung von Impfstoffen in der Schweiz und weltweit zu gewährleisten, müssen Forschungseinrichtungen, Behörden, produzierende, distributive und leistungserbringende Institutionen und Leistungserbringende in einem komplexen und dynamischen System zusammenspielen. Alle Beteiligten agieren in einem internationalen Umfeld und unter Rahmenbedingungen, die oft nur beschränkt beein-

\section{Wichtige Internetadressen für} die Ärzteschaft

www.helvecura.ch/de/arzneimittel-verzeichnis: Aktualisiertes Verzeichnis der für die Pflichtlagerhaltung relevanten Produkte. www.swissmedic.ch/sonderbewilligungen: Sonderbewilligungen für die Beschaffung von Arzneimitteln, die in der Schweiz nicht zugelassen oder nicht lieferbar sind.

www.bwl.admin.ch/meldestelle: Meldestelle für aktuelle Versorgungsengpässe im Heilmittelsektor.

www.infovac.ch: die direkte Informationsstelle für Impffragen. www.sginf.ch: Schweizerische Gesellschaft für Infektiologie www.bag.admin.ch/impfstoffversorgung: Bundesamt für Gesundheit

flussbar und schwer berechenbar sind. Das führt zu vielschichtigen und interdependenten Ursachen für Engpässe, die in einen reduzierten Schutz der Bevölkerung durch Impfungen münden.

Damit die Impfempfehlungen umgesetzt und der Bevölkerungsschutz längerfristig garantiert sowie allfällige Notsituationen und Epidemien bekämpft werden können, sind Liefer- und Versorgungsengpässe wenn immer möglich zu vermeiden. Dies könnte über eine Harmonisierung zwischen Swissmedic und der europäischen Arzneimittelagentur (EMA) angegangen werden. In diesem Zusammenhang wären Zulassung, Erleichterung von Importmöglichkeiten mit geregelter Kostenübernahmen (OKP) bei Engpässen und Weiteres zu thematisieren. Zudem müsste eine mögliche Ausweitung der staatlichen Verantwortung in Bezug auf Beschaffung, Verwaltung und Verteilung zumindest in Betracht gezogen werden. Mit der Ausweitung der Pflichtlagerhaltung auf Impfstoffe ist ein neues und wichtiges Instrument geschaffen worden. Diese Massnahme dürfte jedoch frühestens 2019 zu einer Verbesserung der Versorgungslage führen. Das reicht jedoch nicht, um sich zurückzulehnen. Die konstante Versorgung des Landes mit Impfstoffen muss Ziel sein und weiterhin bleiben. Dies bedingt die Anwendung der bereits heute gesetzlich verankerten Möglichkeiten, die Schaffung von neuen Optionen sowie eine kontinuierliche interprofessionelle Kommunikation betreffend weiterer Schritte.

\footnotetext{
Literatur

1 Strategic Advisory Group of Experts (SAGE) on Immunization, Meeting April 2016

220 von 26 Ländern haben die Umfrage beantwortet
}

Bildnachweis

() Singburi | Dreamstime.com 